\title{
Influência de projetos pedagógicos interdisciplinares na atividade física habitual e no estado nutricional
}

\author{
Carolina Braz Carlan Rodrigues* \\ Karla Mendonça Menezes** \\ Vanessa Candito*** \\ Félix Alexandre Antunes Soares****
}

\section{Resumo}

Investigou-se a influência de projetos interdisciplinares na atividade física e estado nutricional de escolares dos $6 .^{\circ}$ e $7 .^{\circ}$ anos do ensino fundamental de uma escola pública estadual. Os docentes se organizaram em grupos na elaboração de projetos interdisciplinares: "Atividade Física e Lazer" (Grupo A) e "A importância da alimentação saudável" (Grupo B). Para avaliar o estado nutricional e a atividade física habitual dos escolares, utilizaram-se as recomendações da Organização Mundial da Saúde e os pontos de corte do Sistema de Vigilância Alimentar Nutricional. Observaram-se diferenças significativas entre as avaliações na atividade física habitual do Grupo A, enquanto o Grupo B não apresentou alterações. O desenvolvimento de projetos interdisciplinares influenciou positivamente na atividade física habitual, no entanto, não foram percebidas alterações consistentes no estado nutricional dos escolares.

Palavras-chaves: Educação em Saúde. Projetos Interdisciplinares. Ensino Fundamental.

* Universidade Federal de Santa Maria - RS. carolina_carlan@hotmail.com

** Universidade Federal de Santa Maria - RS. karlam.ef@gmail.com

*** Universidade Federal do Rio Grande do Sul. vanecandito@gmail.com

**** Universidade Federal de Santa Maria - RS. felix@ufsm.br 


\section{Interdisciplinary educational project influence on physical activity usual and nutritional status}

\section{Abstract}

The influence of interdisciplinary projects on the physical activity and nutritional status of 6 th and 7 th grade elementary school students at a state public school was investigated. The teachers organized themselves into groups in the elaboration of interdisciplinary projects: "Physical Activity and Leisure" (Group A) and "The importance of healthy eating" (Group B). To assess the nutritional status and habitual physical activity of the students, we used the recommendations of the World Health Organization and the cutoff points of the Nutritional Food Surveillance System. Significant differences were observed between the evaluations in the usual physical activity of Group A, while Group B did not present alterations. The development of interdisciplinary projects positively influenced the habitual physical activity, however, no consistent changes were observed in the nutritional status of the students.

Keywords: Health Education. Interdisciplinary Projects. Elementary School.

\section{Influencia de proyectos pedagógicos interdisciplinarios sobre la actividad física habitual y el estado nutricional}

\section{Resumen}

Se investigó la influencia de los proyectos interdisciplinarios en la actividad física y el estado nutricional de los alumnos de $6 .^{\circ}$ y $7 .^{\circ}$ de primaria en una escuela pública estatal. Los docentes se organizaron en grupos en la elaboración de proyectos interdisciplinarios: "Actividad física y ocio" (Grupo A) y "La importancia de una alimentación saludable" (Grupo B). Para evaluar el estado nutricional y la actividad física habitual de los estudiantes, utilizamos las recomendaciones de la Organización Mundial de la Salud y los puntos de corte del Sistema de Vigilancia Nutricional de Alimentos. Se observaron diferencias significativas entre las evaluaciones en la actividad física habitual del Grupo A, mientras que el Grupo B no presentó alteraciones. El desarrollo de proyectos interdisciplinarios influyó positivamente en la actividad física habitual, sin embargo, no se observaron cambios consistentes en el estado nutricional de los estudiantes. Palabras clave: Educación en salud. Proyectos interdisciplinarios. Enseñanza fundamental. 


\section{Introdução}

A obesidade e o sedentarismo têm atingido proporções preocupantes em todo o mundo atualmente. Os níveis insuficientes de atividade física entre crianças e adolescentes são atribuídos, em grande parte, ao incremento do tempo despendido com recursos eletrônicos e a diminuição das opções de lazer ativo. Nesse sentido, ações educativas podem visar à sensibilização e ou conscientização de problemas de saúde ou ações preventivas, atribuindo aos comportamentos saudáveis significados e interesse dos jovens (HALLAL et al., 2010).

Os Parâmetros Curriculares Nacionais (1997) destacam a importância do conhecimento e o cuidado do corpo, consideram e valorizam a boa alimentação, e reconhecem a relevância da conscientização do sujeito para uma melhor qualidade de vida. Assim a escola tem sido definida como um local adequado para o desenvolvimento de atividades que envolvam a promoção da saúde, medidas educativas e preventivas, abrangendo a participação dos professores como agentes multiplicadores.

Nesse contexto, a promoção da saúde e os temas relacionados à saúde devem ser trabalhados de forma transversal, onde haja a contextualização com os conteúdos e também informações sobre hábitos de vida saudáveis. Para isso, é importante que a consciência crítica do aluno sobre os aspectos da promoção da saúde seja adquirida por meio de um processo educativo. Dessa forma, a formação integral do aluno será possível, e assim terá ferramentas e saberes para tomar decisões adequadas para uma melhor qualidade de vida e da saúde (ILHA et al., 2013).

Para Freire (2011), a participação dos estudantes no seu processo formativo é fundamental, pois dessa forma é possível abarcar aspectos importantes do contexto e da realidade dos envolvidos. Assim, por meio da dialogicidade entre educadores e educandos é possível que as situações relevantes sejam superadas, portanto, transformando a realidade dos sujeitos. Nesse sentido, a interdisciplinaridade perpassa o processo metodológico de construção do 
conhecimento pelo sujeito com base em sua relação com o contexto, a realidade e a cultura.

Nesse sentido, Rossi et al. (2013) destacam que as intervenções relacionadas ao trabalho interdisciplinar, elaboradas pelos professores e oferecidas na escola, devem levar em conta as particularidades de cada educando, a realidade da interação familiar e os educadores envolvidos. Logo, por meio da interdisciplinaridade, a escola proporciona um complemento no conhecimento escolar, já que esta ocorre a partir da interação de duas ou mais disciplinas na troca de saberes e no compartilhamento de ideias. Agregando a interdisciplinaridade como uma ferramenta viabilizadora, que se torna indispensável para a construção de novos saberes, enobrecendo as trocas de vivências e saberes.

O educador, nessa perspectiva, desempenha papel fundamental para organizar as atividades e formular as situações que propiciem a aprendizagem dos conhecimentos de forma significativa (BONATTO et al., 2012). Desse modo, um caminho possível que pode influenciar a relação das disciplinas é o trabalho por meio de projetos, como forma de estratégia para a construção de conhecimentos em conjunto (ROJAS et al., 2013; ARAÚJO, 2003).

Nesse contexto, ao analisar as percepções de professores sobre a implantação de uma proposta de ensino-aprendizagem por projetos, Ilha et al. (2013) ressaltam que quando se fala em desenvolvimento dos projetos de ensino ou projetos de aprendizagem, embora ambos tenham algumas características semelhantes, o projeto de ensino é estruturado e desenvolvido pelo docente, enquanto que nos projetos de aprendizagem o aluno constrói o conhecimento de forma colaborativa e o professor age como mediador da aprendizagem. Em ambos, o ponto de partida é uma situação problemática trazida pelos educandos ou proposta pelo professor, cujo enfrentamento vai requerer a organização de atividades de aprendizagem.

Em complemento, Ribeiro (2008) define a estratégia de projetos como uma metodologia ativa, colaborativa, centrada nos 
estudantes e voltada para uma situação problema. O autor destaca também cinco elementos que considera essenciais para o desenvolvimento dos projetos, sejam eles: de ensino, aprendizagem ou ensino-aprendizagem: colocação de problemas; capacidade dos problemas integrarem conceitos de diversas disciplinas; trabalho em grupo; existência de um processo formal de resolução dos problemas e o estudo independente dos escolares.

A partir dessas considerações, o presente estudo teve como objetivo identificar a influência de projetos pedagógicos interdisciplinares na atividade física habitual e no estado nutricional de escolares dos $6 .^{\circ}$ e $7 .^{\circ}$ anos do ensino fundamental de uma escola pública do interior do Rio Grande do Sul.

\section{Método}

Esse estudo caracteriza-se como um estudo de caso, com enfoques quali-quantitativos (YIN, 2001) e é parte integrante de uma dissertação de mestrado (CARLAN, 2016).

Participaram do estudo 61 escolares dos $6 .^{\circ}$ e $7 .^{\circ}$ anos do ensino fundamental de uma escola da rede pública estadual do interior do Rio Grande do Sul. Foram constituídos dois grupos: o Grupo A compreendeu os escolares do 6. ${ }^{\circ}$ ano e quatro professores, das seguintes disciplinas: educação física, matemática, geografia e português. O Grupo B contemplou os escolares do $7 .^{\circ}$ ano e dois professores das disciplinas de história e inglês.

A sistematização dos projetos desenvolvidos pelos grupos está apresentada no Quadro 1, a seguir: 
Quadro 1 - Estruturação dos Grupos

\begin{tabular}{|c|c|c|}
\hline \multicolumn{2}{|r|}{ Grupo A } & Grupo B \\
\hline $\begin{array}{l}\text { Definição do } \\
\text { tema }\end{array}$ & $\begin{array}{l}\text { O tema "Atividade Física e } \\
\text { Lazer" foi proposto pelos } \\
\text { escolares, a partir de proble- } \\
\text { matizações propostas pelos } \\
\text { professores e da identificação } \\
\text { da carência de áreas de lazer } \\
\text { na comunidade e alto índice } \\
\text { de excesso de peso corporal } \\
\text { entre os estudantes; }\end{array}$ & $\begin{array}{l}\text { O tema "A importância } \\
\text { da alimentação saudável" } \\
\text { surgiu da percepção dos } \\
\text { professores de que os } \\
\text { escolares adotavam uma } \\
\text { alimentação inadequada } \\
\text { na escola e não realiza- } \\
\text { vam o desjejum. }\end{array}$ \\
\hline $\begin{array}{l}\text { Conhecimen- } \\
\text { tos prévios dos } \\
\text { escolares }\end{array}$ & $\begin{array}{l}\text { Por meio das problematiza- } \\
\text { ções, os escolares confron- } \\
\text { taram as opiniões e dúvidas } \\
\text { sobre o tema escolhido e de- } \\
\text { finiram a questão norteadora: } \\
\text { "Quais são as atividades físi- } \\
\text { cas e de lazer praticadas pela } \\
\text { nossa comunidade?" }\end{array}$ & $\begin{array}{l}\text { As professoras procura- } \\
\text { ram conhecer a realida- } \\
\text { de dos alunos referente } \\
\text { às refeições realizadas } \\
\text { durante o dia. Os escola- } \\
\text { res puderam expor suas } \\
\text { dúvidas, conhecimentos } \\
\text { e suas concepções refe- } \\
\text { rentes ao tema. }\end{array}$ \\
\hline $\begin{array}{l}\text { Sistematização } \\
\text { e pesquisa }\end{array}$ & $\begin{array}{l}\text { Com o intuito de responder } \\
\text { ao questionamento norteador } \\
\text { do projeto, os escolares se en- } \\
\text { volveram na construção de } \\
\text { um questionário a ser aplica- } \\
\text { do na comunidade. Para tan- } \\
\text { to, os professores planejaram } \\
\text { e desenvolveram atividades } \\
\text { interdisciplinares (pesquisas } \\
\text { na sala de informática, bi- } \\
\text { blioteca e fora do contexto } \\
\text { escolar), confrontando os } \\
\text { conhecimentos prévios dos } \\
\text { alunos com outras visões e } \\
\text { conhecimentos, analisando } \\
\text {-as e relacionando-as com os } \\
\text { conteúdos específicos de cada } \\
\text { disciplina. }\end{array}$ & $\begin{array}{l}\text { Os professores embasa- } \\
\text { ram suas aulas no livro } \\
\text { didático, por meio da } \\
\text { pirâmide alimentar e de } \\
\text { diversas culturas e culi- } \\
\text { nárias. }\end{array}$ \\
\hline
\end{tabular}




\begin{tabular}{|c|c|c|}
\hline \multicolumn{2}{|r|}{ Grupo A } & Grupo B \\
\hline $\begin{array}{l}\text { Produção do } \\
\text { conhecimento }\end{array}$ & $\begin{array}{l}\text { A partir dos pressupostos ex- } \\
\text { postos durante as aulas, foram } \\
\text { desenvolvidos pelos escolares } \\
\text { os questionários para verificar } \\
\text { as atividades físicas realizadas } \\
\text { pela comunidade, a frequên- } \\
\text { cia; o lugar ideal para praticar } \\
\text { exercícios físicos; se o indiví- } \\
\text { duo se exercitava por lazer ou } \\
\text { para melhorar o condiciona- } \\
\text { mento físico; }\end{array}$ & $\begin{array}{l}\text { A partir das atividades } \\
\text { pedagógicas, os alunos } \\
\text { construíram cartazes } \\
\text { com a pirâmide alimen- } \\
\text { tar de diferentes culturas, } \\
\text { desenvolvendo seus car- } \\
\text { dápios alimentares com } \\
\text { base nela. }\end{array}$ \\
\hline $\begin{array}{l}\text { Resultados do } \\
\text { projeto }\end{array}$ & $\begin{array}{l}\text { Os resultados foram expostos } \\
\text { na mostra pedagógica da es- } \\
\text { cola. Os escolares produziram } \\
\text { materiais informativos sobre a } \\
\text { importância da atividade física } \\
\text { e do lazer, que foram entre- } \\
\text { gues na comunidade durante } \\
\text { uma caminhada organizada } \\
\text { pelos docentes. }\end{array}$ & $\begin{array}{l}\text { Os resultados foram } \\
\text { apresentados na mostra } \\
\text { pedagógica da escola. }\end{array}$ \\
\hline
\end{tabular}

Fonte: Os autores

A atividade física habitual dos escolares foi investigada por meio de um recordatório, com questões previamente estruturadas, que consideraram a duração (minutos por dia) e o tipo de atividade física praticada. O nível de atividade física foi determinado seguindo as recomendações da OMS (2009), a qual afirma que crianças e adolescentes entre 5 a 17 anos de idade devem acumular pelo menos 60 minutos de atividade física moderada ou vigorosa diárias. $\mathrm{O}$ estado nutricional foi avaliado a partir do índice de massa corporal (IMC) e analisado através dos percentis de IMC/Idade, utilizando como referência as curvas da SISVAN (2011). As avaliações foram realizadas no início (pré-teste) e no final (pós-teste) do ano letivo.

O tratamento estatístico foi realizado no SPSS (versão 23). Utilizaram-se testes descritivos e o Teste t de Student para compa- 
rar as variáveis no pré e pós-testes intragrupos e intergrupos. Em todas as análises foi considerado um intervalo de confiança de $95 \%$.

\section{Resultados e discussões}

A atividade física habitual e o estado nutricional de escolares foram investigados antes e após o incremento de projetos interdisciplinares, desenvolvidos durante o ano letivo, como recurso pedagógico.

Conforme detalhado, no Quadro 1 a estruturação e o desenvolvimento dos projetos apresentaram características similares em ambos os grupos. No entanto, vale destacar alguns aspectos importantes que influenciaram a definição do tema e o desenvolvimento dos mesmos. Enquanto no Grupo A o tema "Atividade Física e Lazer" foi proposto pelos escolares, a partir de problematizações propostas pelos professores, o tema contemplado pelo Grupo B, "A importância da alimentação saudável", surgiu da percepção dos professores de que os escolares adotavam uma alimentação inadequada na escola e não realizavam o desjejum. Assim, os projetos interdisciplinares tiveram características de projeto de aprendizagem e projetos de ensino, respetivamente.

Na tabela 1 estão apresentados os resultados relativos à frequência semanal da atividade física referida pelos escolares.

Tabela 1 - Frequência semanal da atividade física habitual

\begin{tabular}{|l|c|c|c|c|}
\hline \multirow{2}{*}{$\begin{array}{l}\text { Frequência } \\
\text { Semanal }\end{array}$} & \multicolumn{2}{|c|}{ Grupo A } & \multicolumn{2}{c|}{ Grupo B } \\
\cline { 2 - 5 } & Pré-teste & Pós-teste & Pré-teste & Pós-teste \\
\hline Até 1 vez & $03,8 \%$ & --- & $05,7 \%$ & $17,1 \%$ \\
\hline 2 a 3 vezes & $34,6 \%$ & $23,1 \%$ & $48,6 \%$ & $28,6 \%$ \\
\hline 4 a 5 vezes & $30,8 \%$ & $19,2 \%$ & $20,0 \%$ & $40,0 \%$ \\
\hline 6 a 7 vezes & $30,8 \%$ & $57,7 \% *$ & $25,7 \%$ & $14,3 \%$ \\
\hline
\end{tabular}

*Diferença significativa entre o pré e pós-teste intragrupo $(p=0,011)$

Fonte: Os autores 
Os resultados demonstraram no pré-teste que o maior percentual de escolares, do Grupo A, indicou a frequência de atividade física na faixa de 2 a 3 vezes na semana, seguidos de 4 a 5 vezes e 6 a 7 vezes na semana. Ao analisar as respostas do pós-teste, foi possível identificar que os escolares ampliaram a periodicidade semanal de suas atividades físicas, em maior concentração de dados na faixa de 6 a 7 vezes na semana. O Grupo B apresentou alterações sutis entre as avaliações, não sendo observadas diferenças estatísticas entre os testes.

Quando comparados os grupos, observou-se que ambos não diferiram significativamente no teste inicial. No entanto, após o desenvolvimento dos projetos, as alterações foram percebidas por meio do aumento na frequência semanal de prática de atividades físicas dos estudantes do Grupo A resultaram em diferença significativa $(p=0,022)$ entre os grupos no pós-teste.

A seguir, na tabela 2 , estão apresentados os valores do estado nutricional.

Tabela 2 - Estado nutricional dos escolares do Grupo A e B

\begin{tabular}{|l|c|c|c|c|}
\hline \multirow{2}{*}{ Estado Nutricional } & \multicolumn{2}{|c|}{ Grupo A } & \multicolumn{2}{c|}{ Grupo B } \\
\cline { 2 - 5 } & $\begin{array}{c}\text { Pré- } \\
\text { teste }\end{array}$ & Pós-teste & Pré-teste & Pós-teste \\
\hline Eutrofia & $64,0 \%$ & $58,3 \%$ & $62,5 \%$ & $62,5 \%$ \\
\hline Acima do peso & $36,0 \%$ & $41,7 \%$ & $37,5 \%$ & $37,5 \%$ \\
\hline
\end{tabular}

Fonte: Os autores

Os resultados demonstraram maior prevalência de escolares classificados em eutrofia, em ambos os grupos, ao longo do estudo. Não foram observadas alterações significativas no estado nutricional dos escolares entre os testes.

Este estudo se propôs a investigar a atividade física habitual e estado nutricional de escolares após o incremento de projetos interdisciplinares, desenvolvidos durante o ano letivo. Em síntese, 
os resultados apontaram mudanças nos hábitos de atividade física habitual nos estudantes que se envolveram com o projeto "Atividade Física e Lazer" (Grupo A). Essas alterações ocorreram principalmente nos estudantes que apresentavam excesso de massa corporal. Em paralelo, os estudantes que se envolveram com o projeto "A importância da alimentação saudável" não apresentaram alterações significativas no estado nutricional e na atividade física habitual.

Machado (2011) enfatiza a importância da prática de atividade física na infância e/ou na adolescência, já que muitos deixam de se exercitar ao longo de seu desenvolvimento biológico e muitas vezes pode contribuir para o desenvolvimento de sobrepeso e também de obesidade.

A mudança de atitudes e de comportamentos nesse processo é gradual, porém o desenvolvimento do projeto "Atividade Física e Lazer" com os educandos do Grupo A pode ter influenciado no aumento da prática de atividade física (pré-teste: 30,8\% pós-teste: $57,7 \%$ ), pois os participantes foram incentivados a conhecer e compreender os benefícios da prática de atividade física, por meio das atividades desenvolvidas pelos educadores. Nesse sentido, o Ministério da Saúde (2002) reforça que a escola pode inferir e orientar os estudantes quanto aos valores e atitudes por meio de estratégias e metodologias, por exemplo, a proposta desenvolvida nesse estudo, a fim de que incentivem a assumir hábitos de vida mais saudáveis.

Para Prochaska et al. (1992), existem seis estágios para a mudança de comportamento: o primeiro é o estágio pré-contemplativo: em que o indivíduo não faz nem pensa em fazer atividade física; o seguinte é o estágio contemplativo: que o indivíduo não faz atividade física, mas pensa em começar a fazer; e o terceiro é o preparação: em que se iniciam algumas mudanças; o quarto é o estágio de ação: no qual o indivíduo faz atividade física regularmente; o próximo estágio é o da manutenção: que dá continuidade ao trabalho iniciado com o estágio de ação; e por último a recaída: que em algum momento existe uma falha na manutenção e ocorre o retorno a qualquer um dos estágios anteriores. Associando os resulta- 
dos do presente estudo aos seis estágios, o Grupo A aumentou significativamente a frequência semanal da prática de atividade física entre o pré e pós-teste $(\mathrm{p}=0,011)$. culminando no estágio de ação. No entanto, os escolares do Grupo B não demonstraram melhora na prática de atividade física em ambos os testes, ficando entre o estágio pré-contemplativo e contemplativo. Contudo, deve-se ressaltar que os estágios de manutenção e recaída fazem parte de um processo com o intuito de uma mudança comportamental, e dessa forma mesmo que o sujeito esteja em um desses estágios ainda poderá perpassar pelos outros, tornando assim um processo cíclico.

A partir dos resultados do estado nutricional, observou-se que não houve melhora para ambos os grupos analisados nos testes aplicados. Muito embora os escolares do Grupo A estivessem praticando mais atividade física, o índice de massa corporal não evidenciou diferença. Ressalta-se que os estudantes do Grupo A foram estimulados a mudarem seus hábitos em relação à atividade física, enquanto que o Grupo B preocupou-se com o desjejum dos educandos pela manhã e a alimentação saudável. Entretanto, é importante frisar, para que ocorram mudanças no estado nutricional, a criança e/ou o adolescente necessitam modificar seus hábitos alimentares, adquirindo uma alimentação balanceada, bem como, a prática regular de exercícios físicos (CELESTRINO; COSTA, 2006).

Contudo, compreende-se que a avaliação do estado nutricional permite monitorar as modificações do crescimento e das mudanças nos indivíduos, porém essas modificações dependem de multifatores como: genéticos, metabólicos, ambientais, comportamentais sociais e culturais, assim como a questão nutricional e a frequência da atividade física, anteriormente mencionados (PINTO; BERNARDI; RAVAZZANI, 2011; GOULART, 2010). Posto isso, percebeu-se que os alunos dos Grupos A e B não obtiveram melhora, pois somente a partir das mudanças dos fatores, citados anteriormente, é que se poderá obter alguma alteração no estado nutricional.

Deste modo, o projeto do Grupo B, desenvolvido com o intuito de alertar para a importância do desjejum pela manhã e para 
uma alimentação balanceada, torna-se relevante, pois pode auxiliar a reduzir e também controlar o peso. Triches e Giuglianib (2005) apontam que a omissão da primeira refeição do dia está relacionada à obesidade, e que o café da manhã pode regular o peso corporal, pois os indivíduos que omitem essa refeição tendem a apresentar uma ingestão inadequada de nutrientes, com índice de massa corporal elevado.

Todavia, é importante ressaltar que os educandos participantes dos grupos (A e B) são crianças e adolescentes, e esses seguem os padrões alimentares reproduzidos pelos pais e familiares em suas residências. Portanto, mesmo que as intervenções, implementações e projetos ocorram no ambiente escolar, e sejam direcionados para a melhoria da promoção da saúde, visando à alimentação balanceada, deve-se considerar que se os hábitos alimentares não forem modificados também no ambiente familiar, e não ocorrerão modificações no estado nutricional (MELLO; LUFT; MEYER, 2004). Logo, uma educação direcionada ao conhecimento nutricional deve ser iniciada na infância, para que assim a escola possa dar seguimento no processo educativo, a fim de auxiliar na prevenção do sobrepeso e obesidade.

Barreto et al. (2005) frisam que o desenvolvimento dos trabalhos pedagógicos, direcionados no sentido da promoção de saúde, visa à conscientização da necessidade da prática de atividade física e de uma alimentação equilibrada, na qual se tornam fundamentais para a diminuição dos índices de sobrepeso e obesidade, assim como o sedentarismo em escolares. Dessa forma, os trabalhos desenvolvidos na escola pelos dois grupos decorreram da observação dos altos índices de obesidade e da alimentação inadequada. E assim foram planejados projetos interdisciplinares, que conduziram $41,7 \%$ dos alunos do Grupo A que se encontravam acima do peso ideal a praticarem mais atividade física. Nesse sentido, os projetos desenvolvidos em ambos os grupos podem ter incentivado os alunos para o aumento da prática de atividade física regular, assim como adquirirem uma alimentação mais saudável. Mas as modifi- 
cações foram visíveis apenas na atividade física, visto que o estado nutricional necessita de um período maior para modificar-se, e também pela forte influência dos multifatores citados.

Desse modo, é possível que os educandos do Grupo A tenham obtidos resultados mais significativos, pelo maior envolvimento em todo o processo educativo. Embora a temática do Grupo B tenha sido pertinente à realidade encontrada dos educandos, se o empenho dos mesmos desde a escolha do tema e planejamento do projeto fosse mais abrangente, os resultados obtidos poderiam ter sido mais expressivos.

Sendo assim, percebe-se a relevância do desenvolvimento de projetos interdisciplinares no ambiente escolar a longo prazo, com o intuito de se promover a saúde. Pois a escola pode auxiliar na construção de um estilo de vida e hábitos saudáveis dos educandos. E a interdisciplinaridade tem um papel importante na construção do conhecimento, pois as práticas pedagógicas são construídas pela coletividade, distanciando-se, assim, da visão fragmentada no processo.

Deste modo, os professores participantes elaboraram e planejaram de forma coletiva, com o auxílio dos escolares para a culminância dos projetos. Foram realizadas atividades disciplinares e interdisciplinares, respeitando as especificidades das distintas áreas, mas valorizando a troca de saberes e experiências nas atividades que possibilitaram a interação dos educandos e educadores. Corroborando, Magoga (2017) declara que a interdisciplinaridade deve ser compreendida como um processo de construção coletiva, em que cada disciplina tem sua importância, de modo que as disciplinas dialoguem em torno do tema de estudo, e problematizem cada uma na sua especificidade.

\section{Considerações finais}

A partir da análise dos resultados, foi possível concluir que muitos escolares do Grupo A aumentaram a prática de atividade física semanal, porém não obtiveram mudanças em seus estados nutricionais. Pode-se referir que esses achados sejam decorrentes do 
projeto realizado pelas educadoras com enfoque temático "Atividade Física e Lazer". No Grupo B não foram encontradas mudanças em relação ao estado nutricional e frequência semanal de atividade física, mesmo com a participação no projeto da importância da alimentação saudável.

A temática do Grupo A foi originada a partir do consenso entre professores e estudantes no processo do projeto, enquanto no Grupo B o tema norteador do projeto surgiu pela observação dos professores acerca da má alimentação dos educandos pela manhã. É possível que o maior envolvimento dos participantes do Grupo A seja decorrente do interesse e motivação pela temática abordada e, sobretudo, tenha surgido dos próprios integrantes. Por conseguinte, ocorreu a melhora nos resultados, onde os escolares se tornaram mais ativos.

Os projetos interdisciplinares podem ter auxiliado na aquisição e mudanças de alguns hábitos dos alunos, porém para que ocorra uma melhora significativa no estado nutricional, é necessário compreender todos os fatores que permeiam o desenvolvimento do sujeito. É importante enfatizar que as mudanças comportamentais são mais rápidas, ao passo que as biológicas dependem de vários fatores para que ocorram modificações ao longo do tempo.

Embora os resultados ainda evidenciem um elevado índice de alunos acima do peso e sedentários, percebeu-se a necessidade de implementação e intervenções de programas, projetos e trabalhos pedagógicos direcionados à saúde no contexto escolar, a fim de desenvolver, influenciar e ressignificar o processo educativo, com o seguimento e culminância desses trabalhos para prevenir futuramente possíveis doenças e que auxiliem na aquisição de hábitos saudáveis. Por meio de ações, como este estudo, é possível que a educação em saúde oportunize espaços de discussão sobre hábitos de vida saudáveis, contribuindo dessa forma para a prevenção de comportamentos de risco, e que assim auxilie também o indivíduo a ponderar suas escolhas, incentivando-o para a sua maior autonomia e responsabilidade com a vida. 


\section{Referências}

ARAÚJO, U. F. Temas transversais e a estratégia de projetos. São Paulo: Moderna, 2003.

BARRETO, S. M.; PINHEIRO, A. R. de O.; SICHIERI, R.; MONTEIRO, C. A.; FILHO, M. B.; SCHIMIDT, M. I.; LOTUFO, P.; ASSIS, A. M.; GUIMARÃES, V.; RECINE, E. G. I. G.; VICTORA, C. G.; COITINHO, D.; PASSOS, V. M. de A. Análise da estratégia global para alimentação, atividade física e saúde, da Organização Mundial da Saúde. Epidemiol. Serv. Saúde, Brasília, v. 14, n. 1, mar. 2005.

BONATTO, A.; BARROS, C. R.; GEMELI, R. A.; LOPES, T. B.; FRISON, M. D. Interdisciplinaridade no ambiente escolar. Seminário de Pesquisa em Educação da Região Sul - IX ANPED SUL, 2012.

BRASIL. Secretaria de Educação Fundamental. Parâmetros curriculares nacionais: introdução aos parâmetros curriculares nacionais/Secretaria de Educação Fundamental. - Brasília: MEC/SEF, 1997.

- Ministério da Saúde - Projeto Promoção da Saúde - Secretaria de Políticas de Saúde. A promoção da saúde no contexto escolar. Revista Saúde Pública 2002; 36(2): 533-5.

Ministério da Saúde. Secretaria de Atenção à Saúde. Departamento de Atenção Básica. Orientações para a coleta e análise de dados antropométricos em serviços de saúde: Norma Técnica do Sistema de Vigilância Alimentar e Nutricional - SISVAN, 2011. Disponível em: http://bvsms.saude. gov.br/bvs/publicacoes/marco_referencia_vigilancia_alimentar.pdf Acesso em: 19/07/2019

CARLAN, C. B. Influência de projetos pedagógicos interdisciplinares na atividade física habitual e no estado nutricional de escolares do ensino fundamental. [Dissertação]. Porto Alegre, UFRGS; 2016.

CELESTRINO, J. O.; COSTA, A. S. A prática de atividade física entre escolares com sobrepeso e obesidade. Revista Mackenzie de Educação Física e Esporte - 2006, 5 (especial): 47-54.

FREIRE, P. Pedagogia do oprimido. 22. ed. Rio de Janeiro: Paz e Terra, 2011.

GOULART, K. T. Sobrepeso e obesidade: implicações e alternativas no contexto escolar [Dissertação]. Santa Maria, UFSM; 2010. 
HALLAL, P. C.; KNUTH, L. G.; CRUZ, D. K. A.; MENDES, M. I.; MALTA, D. C. Physical activity practice among brasilian adolescents. Ciênc. Saúde Coletiva, 15 (2): 3035-3042, 2010.

ILHA, P. V.; LIMA, A. P. S.; WOLLMANN, E. M.; KRUG, M. R.; ROSSI, D. S.; SOARES, F. A. A. Percepções dos professores sobre implantação de uma proposta de aprendizagem por projetos. In: XVII Seminário Internacional de Educação, 2013, Cachoeira do Sul. ANAIS DO XVII SIEDUCA (LIVRO ELETRÔNICO): Aprendente e Ensinante: o mundo nos chama, as relações nos educam. Cachoeira do Sul: IN BOOKS, 2013. p. 134-139.

MACHADO, Y. L. Sedentarismo e suas Consequências em Crianças e Adolescentes. [TCC]. Muzambinho, IFSULMINAS; 2011.

MAGOGA, T. F. Abordagem temática na educação em ciências: um olhar à luz da epistemologia fleckiana. 2017. p. 167. [Dissertação] Santa Maria: UFSM, 2017.

MELLO, E. D.; LUFT, V. C.; MEYER, F. Obesidade infantil: como podemos ser eficazes? Jornal de pediatria (Rio de Janeiro), 80 (3), Maio/Junho, 2004.

PINTO, C. K. de O.; BERNARDI, S. D.; RAVAZZANI, E. D. do A. A influência do desjejum no peso corporal de crianças e adolescentes. [Internet] Curitiba, 2011. Disponível em: http://www.unibrasil.com.br/pdf/nutricao/2011-2/13_tcc. pdf Acesso em: 09/04/2019.

PROCHASKA, J.O.; NORCROSS, J.C.; FOWLER, J.L.; FOLLICK, M.; ABRAMS, D. B. Attendance and outcome in awork site weight control program: processes and stages of changes as process and prediction variables. Addictive Behaviors. Oxford, 17:35-45, 1992.

RIBEIRO, L. R. de C. Aprendizagem baseada e problemas (PBL): uma experiência no ensino superior. São Carlos: EdUFSCar, 2008.

ROJAS, J. S.; PEREIRA, S. R. S.; SILVA, N. L. C.; HAMMES, C. C. Interdisciplinaridade e pesquisa: um encontro de saberes necessários à prática pedagógica na educação infantil. XI Congresso de Nacional de Educação - EDUCERE, 2013.

ROSSI, D. S.; KRUG, M. R.; ILHA, P. V.; LIMA, A. P. S.; SOARES, F. A. A. Imagem corporal, aspectos nutricionais e atividade física em estudantes de uma escola pública. Revista Adolescência e Saúde, Rio de Janeiro, 10 (3): 36-44, jul/set 2013 . 
TRICHES, R. M.; GIUGLIANIB, E. R. J. Obesidade, práticas alimentares e conhecimentos de nutrição em escolares. Revista Saúde Pública, 39 (4): 541-7, 2005.

YIN, R. K. Estudo de caso - planejamento e métodos. 2. ${ }^{\mathrm{a}}$ ed. Porto Alegre: Bookman; 2001.

WORLD HEALTH ORGANIZATION. Global Recommendations Populations of Activity for Health. [Internet] Geneva: WHO; 2010. Disponível em: http://whqlibdoc.who.int/publications/2010/9789241599979_eng.pdf Acesso em: 29/04/2019

\section{Carolina Braz Carlan Rodrigues}

https://orcid.org/0000-0001-7115-0987

Universidade Federal de Santa Maria

Doutoranda no Programa de Pós-Graduação Educação em Ciências: Química da Vida e Saúde na Universidade Federal de Santa Maria - RS.

Contato principal para correspondência.

\section{Karla Mendonça Menezes}

https://orcid.org/0000-0002-7482-0648

Universidade Federal de Santa Maria

Doutoranda no Programa de Pós-Graduação Educação em Ciências: Química da Vida e Saúde na Universidade Federal de Santa Maria - RS.

\section{Vanessa Candito}

https://orcid.org/0000-0003-4663-9590

Universidade Federal do Rio Grande do Sul

Mestranda no Programa de Pós-Graduação Educação em Ciências: Química da Vida e Saúde na Universidade Federal do Rio Grande do Sul - RS.

\section{Félix Alexandre Antunes Soares}

https://orcid.org/0000-0002-6453-7902

Universidade Federal de Santa Maria

Professor Adjunto do Centro de Ciências Naturais e Exatas da Universidade Federal de Santa Maria - RS.

Submetido em: 20-8-2019

Aceito em: 11-11-2019 